\title{
Philosophiques
}

\section{Fonctionnalisme et similarité phénoménale}

\section{Paul Bernier}

Volume 27, numéro 1, printemps 2000

Le matérialisme contemporain

URI : https://id.erudit.org/iderudit/004902ar

DOI : https://doi.org/10.7202/004902ar

Aller au sommaire du numéro

Éditeur(s)

Société de philosophie du Québec

ISSN

0316-2923 (imprimé)

1492-1391 (numérique)

Découvrir la revue

Citer cet article

Bernier, P. (2000). Fonctionnalisme et similarité phénoménale. Philosophiques, 27(1), 99-114. https://doi.org/10.7202/004902ar

\section{Résumé de l'article}

Dans la foulée de divers arguments antiphysicalistes visant à montrer que les qualia ne sont pas fonctionnalisables, Ned Block a proposé un autre argument de ce type, qui repose sur son expérience de pensée de la Terre inversée. L'argument de Block montrerait qu'un sujet peut avoir deux expériences de couleur du même type " phénoménal " qui seraient de deux types fonctionnels distincts puisque, selon lui, elles auraient des contenus intentionnels distincts. Il existerait donc une différence fondamentale entre le contenu phénoménal des expériences sensorielles et leur contenu intentionnel et, dans la mesure où le contenu phénoménal ne pourrait être fonctionnalisé, le fonctionnalisme serait ainsi réfuté, à tout le moins en ce qui concerne les expériences sensorielles. Je soulève une objection qui, si elle est correcte, montre que la distinction que Block tente d'établir entre le contenu phénoménal et le contenu intentionnel des expériences est incohérente.
Ce document est protégé par la loi sur le droit d'auteur. L’utilisation des services d’Érudit (y compris la reproduction) est assujettie à sa politique d'utilisation que vous pouvez consulter en ligne.

https://apropos.erudit.org/fr/usagers/politique-dutilisation/ 


\title{
Fonctionnalisme et similarité phénoménale
}

\author{
PAUL BERNIER \\ bernierp@umoncton.ca \\ Université de Moncton
}

\begin{abstract}
RÉSUMÉ. - Dans la foulée de divers arguments antiphysicalistes visant à montrer que les qualia ne sont pas fonctionnalisables, Ned Block a proposé un autre argument de ce type, qui repose sur son expérience de pensée de la Terre inversée. L'argument de Block montrerait qu'un sujet peut avoir deux expériences de couleur du même type «phénoménal » qui seraient de deux types fonctionnels distincts puisque, selon lui, elles auraient des contenus intentionnels distincts. Il existerait donc une différence fondamentale entre le contenu phénoménal des expériences sensorielles et leur contenu intentionnel et, dans la mesure où le contenu phénoménal ne pourrait être fonctionnalisé, le fonctionnalisme serait ainsi réfuté, à tout le moins en ce qui concerne les expériences sensorielles. Je soulève une objection qui, si elle est correcte, montre que la distinction que Block tente d'établir entre le contenu phénoménal et le contenu intentionnel des expériences est incohérente.
\end{abstract}

\begin{abstract}
Following the popular trend of antiphysicalist arguments which aim at showing that qualia cannot be functionalized, Ned Block has suggested yet another antiphysicalist argument, on the basis of his Inverted Earth thought experiment. The purpose of his argument is to show that a subject could have two color experiences which are of the same phenomenal type but of different functional types, insofar as they have different intentional contents. This argument would support the idea that there exists a substantial distinction between the phenomenal content of sensory experiences and their intentional content, and that functionalism is false, at least with respect to sensory experiences. I raise an objection to Block's argument which, if correct, shows that the distinction he proposes between the phenomenal and the intentional content of sensory experiences is incoherent.
\end{abstract}

\section{Introduction}

Selon la thèse fonctionnaliste, les états mentaux sont caractérisés par leurs rôles causaux relativement aux stimuli, aux autres états mentaux, et aux comportements. Pour un très grand nombre de philosophes de l'esprit la thèse fonctionnaliste constitue une solution physicaliste adéquate au problème cerveau-esprit, à tout le moins pour ce qui concerne les états mentaux intentionnels, comme les croyances et les désirs ${ }^{1}$. M ais dans le cas de la conscience phénoménale, ou ce qu'on a appelé les « qualia », la situation est très différente. Commele note Thomas $\mathrm{N}$ agel au tout début de son célèbre article

1. Voir notamment Armstrong, D., A M aterialist Theory of M ind, London, Routledge \& Kegan Paul, 1968 ; Lewis, D., "An A rgument for the Identity Theory », Journal of Philosophy, 67, 1970, p. 203-211; et Kim, J., "Émergence, modèles de réduction, et le mental », dans le présent numéro, p. 9-24. 
«W hat Is It Like To Be a Bat? », « la conscience est ce qui rend le problème cerveau-esprit intraitable ${ }^{2} »$. Bien que cette idée de $N$ agel n'ait pas été universellement admise, dans la littérature récente, elle a eu une influence considérable, et un grand nombre de philosophes l'ont endossée sur la base de divers arguments ${ }^{3}$. Si l'un ou l'autre de ces arguments était effectivement fondé, cela montrerait que le fonctionnalisme n'est pas une solution généralement adéquate au problème cerveau-esprit, dans la mesure où les qualia résisteraient à une analyse fonctionnelle.

$M$ on objectif est de défendre le fonctionnal isme contre un argument qui a récemment été formulé par $\mathrm{N}$ ed Block. Comme je le précise dans ce qui suit, cet argument est une variation sur le thème du célèbre argument du spectre inversé. $M$ on objectif est donc plutôt modeste puisque si ma réfutation était correcte, elleaurait une portée relativement restreinte comptetenu de la diversité des arguments antiphysicalistes qui ont été suggérés pour montrer que les qualia, ou la conscience phénoménale, ne pourraient être fonctionnalisés. $M$ ais cette discussion me paraît tout de même importante car si nous comprenons clairement pourquoi l'argument de Block est erroné, cela devrait nous placer dans une bonne position pour défendre le fonctionnalisme de façon générale et pour comprendre ce qui neva pas avec les divers arguments visant à montrer qu'il serait impossible de fonctionnaliser les qualia.

\section{L'inversion intrapersonnelle}

Dans un article récent ${ }^{4}, \mathrm{~N}$ ed Block a soutenu que même si nous devions accepter que les expériences sensorielles - par exemplel'expérience visuellequej'ai Iorsque je suis confronté visuellement à une tomate dans des conditions d'éclairage normales - ont un contenu intentionnel qui est fonctionnalisable,

2. Nagel, T., «What Is It Like To Be a Bat?», dans Block, N., Flanagan, O. et Güzelderde G., dir., The N ature of C onsciousness, Cambridge (M ass.), M IT Press, 1997, p. 519 (paru initialement dans Philosophical Review, 83, 1974, p. 435-450).

3. Je pense principalement à l'argument des qualia absents, à l'argument de la connaissance de Frank Jackson, à l'argument du spectre inversé et à celui du fossé explicatif (explanatory gap). Voir respectivement Block, N., «Troubles W ith Functionalism », dans Block, N., dir., Readings in Philosophy of Psychology, vol. 1, Cambridge (M ass.), H arvard University Press, 1980 ; Jackson, F., « Epiphenomenal Q ualia », Philosophical Q uarterly, 32, 1982, p. 127-136 ; Shoemaker, S., "The Inverted Spectrum », Journal of Philosophy, 74, 1981, p. 357-381 ; Levine, J., " M aterialism and Q ualia : The Explanatory Gap », Pacific Philosophical Q uarterly, 64, 1983, p. 354-361. II existe bien sûr des différences importantes dans la portée respective de ces arguments, mais tous visent à poser une difficulté de principe au physicalisme ou, de façon générale, au projet de naturalisation de l'esprit, et particulièrement à la thèse fonctionnaliste.

4. Block, N., "Inverted Earth», dans Tomberlin, J., dir., Philosophical Perspectives, vol. 4, A tascaredo, Ridgeview, 1990, p. 31-52 ; réédité dans Block, Flanagan et Güzelderde, dir., The $\mathrm{N}$ ature of Consciousness, p. 677-693. N os références ultérieures à ce texte renvoient à cette dernière édition. 
cela ne suffirait pas à résoudre le problème de la conscience phénoménale puisque, selon lui, les expériences sensorielles auraient en outre un contenu purement phénoménal, ou qualitatif, qui ne pourrait être fonctionnalisé. Un des principaux objectifs de l'argument de Block est donc d'établir une distinction fondamentale entre le contenu phénoménal des expériences sensorielles et leur contenu intentionnel. Si mes objections dans ce qui suit sont correctes, alors cette distinction est trompeuse, et elle devrait être rejetée.

Dans un premier temps, il est utile de faire quelques remarques générales à propos de la principale motivation de l'argument de Block qui s'inscrit dans le cadre d'une discussion de l'argument du spectre inversé. En effet, Block croit que ce dernier, dans sa version intrapersonnellestandard qui a été suggérée par Sydney Shoemaker, serait sujet à certaines objections d'incohérence ${ }^{5}$. M ême s'il n'est pas tout à fait clair qu'il tienne ces objections pour décisives, il suggère que son propre argument, soit l'argument de la Terre inversée, est préférable à celui du spectre inversé dans la mesure où, selon lui, ce type d'objections d'incohérence ne pourrait lui être adressé. Comme on le sait, l'argument du spectre inversé cherche à montrer qu'une individuation des expériences de couleur qui repose uniquement sur leur rôle fonctionnel ou sur les dispositions comportementales des sujets serait inadéquate dans la mesure où il serait possible d'imaginer que deux expériences de couleur puissent avoir des contenus phénoménaux différents alors qu'elles seraient du même type fonctionnel. Et, comme nous le verrons, l'argument de Block repose sur une expérience de pensée qui vise à établir que deux expériences de couleur pourraient avoir le même contenu phénoménal, alors qu'elles seraient de types fonctionnels différents.

Sydney Shoemaker a soutenu que si nous devons admettre la cohérence de l'argument du spectre inversé dans sa version interpersonnelle, c'est-àdire l'idée que deux sujets puissent avoir des expériences phénoménalement inverses qui seraient du même type fonctionnel, alors celui-ci doit reposer sur la cohérence d'une version intrapersonnelle de cette idée. A utrement dit, la plausibilité de l'argument ne peut reposer uniquement sur des considérations relevant del'attribution en troisième personne de prédicats comme « $\mathrm{x}$ paraît bleu » ou «x paraît être de la même couleur que y ». Selon Shoemaker, la cohérence d'une telle possibilité doit reposer sur le point de vue de la première personne. En effet, si la signification d'un prédicat comme " $x$ paraît bleu » était uniquement déterminée par les dispositions linguistiques des sujets à l'appliquer dans différentes circonstances de stimulation, alors l'idée

5. Voir Block, «Inverted Earth», p. 681-682. Pour la version intrapersonnelle standard de l'argument du spectre inversé, voir Shoemaker, « The Inverted Spectrum ». Pour lesdites « objections d'incohérence », voir Dennett, D., «Q uining Q ualia », dans Lycan, W., dir., M ind and Cognition, Oxford, Blackwell, 1990, p. 519-548; et Rey, G., M ind Without Consciousness: A D iscrepancy Between Explanatory and M oral Psychology, Cambridge (M ass.), M IT Press, à paraître. 
centrale de l'argument du spectre inversé serait inintelligible, à savoir par exemple que ce qui paraît bleu à Pierre paraît jaune à J ean (et inversement), alors que ce qui paraît bleu à Pierre produit chez lui un état mental du même type fonctionnel que celui produit chez J ean par ce qui lui paraît jaune. Car si c'était le cas, cela reviendrait à dire les deux choses suivantes, qui sont conjointement incohérentes : i) Pierre est disposé à dire « ceci paraît bleu » dans les circonstances où Jean est disposé à dire « ceci paraît jaune », et ii) dans ces circonstances, J ean et Pierre ont des états mentaux du même type fonctionnel. Et, bien évidement, compte tenu de leurs dispositions à produire deux énoncés différents, leurs états mentaux respectifs devraient être catégorisés comme étant de deux types fonctionnels distincts.

L'idée d'une inversion intrapersonnelle peut être résumée de la façon suivante, en quatre étapes ${ }^{6}$. Premièrement, on imagine un sujet qui est tout à fait normal. À la deuxième étape, on suppose que des lentilles qui ont la propriété d'inverser le spectre des couleurs sont insérées sous la cornée du sujet qui aurait alors l'impression que les couleurs sont inversées. Par exemple, à cette étape, le sujet serait disposé à dire « Les tomates mûres me paraissent vertes » et « L'herbe me paraît rouge ». Évidemment, cela est insuffisant pour appuyer la thèse du spectre inversé puisque son expérience, disons de rouge, changerait du même coup de rôle fonctionnel. M ais l'idée est qu'après une certaine période d'adaptation pendant laquelle le sujet utiliserait les prédicats de couleur de façon confuse, il pourrait éventuellement les utiliser de façon conforme à l'usage intersubjectif admis dans sa communauté, et cela malgré l'inversion de son spectre. La troisième étape est cruciale puisque le sujet doit alors être en mesure de poser des jugements de similarité comme le suivant :

(1) Les tomates mûres me paraissent être de la même couleur que l'herbe me paraissait être jadis.

Le but de cette étape est de donner une plausibilité, sur la base d'intuitions en première personne, à l'idée que ce que cela fait au sujet que d'être confronté visuellement à des tomates mûres, maintenant, est différent de ce que cela lui faisait jadis, et par conséquent que le contenu de son expérience phénoménale est distinct de celui de l'expérience phénoménale d'un sujet «normal » qui se trouverait dans les mêmes conditions stimulatoires. C ependant, cela est encore insuffisant pour garantir la cohérence de la thèse du spectre inversé, dans la mesure où le sujet aurait encore des dispositions " déviantes », comme celle à énoncer (1), ce qui suffit pour nier que son expérience de couleur soit du même type fonctionnel que celui des expériences que nous, sujets normaux, aurions dans les mêmes conditions stimulatoires. $M$ ais cette troisième étape est cruciale car elle en appelle à l'idée que le sujet jouit d'une certaine autorité épistémique le justifiant à croire et nous

6. Je reprends ici, pour l'essentiel, la présentation de Block dans «Inverted Earth», p. 681-682. 
incitant à accepter, que ce que cela lui fait que d'être confronté visuellement à une tomate mûre maintenant est différent de ce que cela lui faisait jadis, même si cette étape est insuffisante pour établir l'identité fonctionnelle d'états mentaux phénoménalement distincts.

Finalement, à la quatrième étape, nous supposons que le sujet utilise les prédicats de couleur de façon tout à fait conforme à l'usage intersubjectif. Pour cela, c'est-à-dire pour éviter que le sujet ait la disposition « déviante » à produire des énoncés comme (1), nous devons supposer qu'il serait désormais affligé d'une amnésie d'un type très spécifique, en raison de laquelle il aurait oublié tout ce qui lui est arrivé avant l'insertion des lentilles inversantes. Donc, si nous acceptons que l'inversion a bel et bien eu lieu, c'est-à-dire si nous donnons foi aux jugements de similarité que le sujet était disposé à faire à la troisième étape avant que l'amnésie ne se produise, alors nous serions en présence d'une inversion intrapersonnelle du spectre, dans la mesure où rien ne nous contraint, logiquement, à accepter que, suite à l'amnésie, le spectre devrait nécessairement se ré-inverser à nouveau pour redevenir «normal ». Ce que cela lui fait que d'être visuellement confronté à des tomates mûres à la quatrième étape est différent de ce que cela lui faisait avant l'inversion des lentilles, alors que ces deux expériences auraient exactement le même rôle fonctionnel. La troisième étape met en évidence l'intuition en première personne selon laquelle ce que cela lui fait, à la quatrième étape, d'être confronté visuellement à des tomates mûres est différent de ce que cela nous fait. Et, l'hypothèse d'amnésie garantit que ses expériences et les nôtres sont bien du même type fonctionnel puisqu'à la quatrième étape le sujet ne serait plus disposé à faire des énoncés comme (1). Compte tenu de son amnésie, ses dispositions seraient conformes aux nôtres. C'est ainsi que la plausibilité d'une inversion intrapersonnelle permet de rendre intelligible I'inversion interpersonnelle.

$M$ ais, comme Block le fait remarquer, certains philosophes se sont objectés à cet argument en proposant que le jugement de similarité en première personne qui est requis à la troisième étape est incohérent, ou bien parce que des jugements de ce type devraient être interprétés comme indiquant que le sujet utilise correctement les prédicats de couleurs mais qu'il a certains problèmes de mémoire, ou sinon que le sujet ne comprend tout simplement pas la signification des prédicats de couleur qu'il utiliserait de façon confuse. II ne me paraît pas évident queces objections soient dirimantes, et mon but ici n'est pas de les évaluer mais simplement de rappeler ce qui semble êtrela principale motivation de l'argument de Block. En effet, Block croit que ce type d'objection d'incohérence ne pourrait tout simplement pas être adressé à son propre argument. Et c'est précisément pour cette raison qu'il suggère que son argument doit être préféré à l'argument standard du spectre inversé. $M$ ais si ma réfutation de l'argument de Block est correcte, c'est plutôt celui-ci qui est confrontéà une objection d'incohérence. Cela dit, la question de savoir si les considérations que je propose pour réfuter l'argument de Block pourraient servir 
de base à une objection de l'argument standard du spectre inversé est une question ouverte que je ne pourrai aborder dans cet article $e^{7}$.

\section{L'argument de Terre inversée}

L'argument de Block repose sur l'expérience de pensée suivante ${ }^{8}$. Supposons qu'il existe une planète, Terre inversée, qui diffère de la Terre uniquement des deux façons suivantes. Premièrement, les objets sur Terre inversée ont exactement les couleurs complémentaires de leurs contreparties sur la Terre. Par exemple, sur Terre inversée, les tomates mûres sont vertes, l'herbe est rouge, le ciel est jaune, etc. Block souligne que cette supposition repose sur une certaine conception réaliste des couleurs, à savoir que ce sont les objets euxmêmes qui ont ces couleurs bizarres ${ }^{9}$. D euxièmement, les habitants de Terre inversée qui sont les contreparties des gens qui parlent le français sur la Terre parlent ce qu'on pourrait appeler le français inversé. Le français inversé est homo phonique avec le français, mais il en diffère uniquement en ce sens que les prédicats de couleur y sont inversés par rapport aux prédicats de couleur en français. C'est-à-dire que les locuteurs du français inversétraduiraient leur prédicat « rouge » par notre prédicat « vert » et ainsi de suite pour les autres prédicats de couleur. A lors que nous sommes disposés à appliquer le prédicat « rouge » aux tomates mûres sur Terre ainsi qu'à I'herbe sur Terre inversée, les locuteurs du français inversé seraient disposés à l'appliquer aux tomates mûres de Terre inversée et à l'herbe sur Terre. En somme, l'expérience de pensée repose sur la possibilité d'une double inversion, soit une inversion de la couleur des objets et une inversion de la dénotation des prédicats de couleur. Évidemment, ces deux inversions se trouvent en quelque sorte à s'annuler mutuellement, en ce sens que les locuteurs du français inversé accepteraient, sur Terre inversée, les énoncés suivants : "Le ciel est bleu », "L'herbe est verte » et ainsi de suite, tout comme nous les acceptons sur Terre.

7. Cette question se pose dans la mesure où l'argument standard du spectre inversé suggère également la possibilité que le sujet ait deux expériences d'un même type phénoménal mais de types fonctionnels différents, même si lorsqu'on parle du « spectre inversé » on entend généralement la possibilité inverse, à savoir celle de deux expériences de types phénoménaux distincts mais d'un même type fonctionnel. J eremercie un commentateur anonyme d'avoir attiré mon attention sur ce point. Une discussion élaborée de la première de ces possibilités, dans le cadre de l'argument standard du spectre inversé, irait au-delà des limites du présent article.

8. Ibid., p. 682-685.

9. Comme il le note : «I mean everything really has these oddball colors. If you visited Inverted Earth along with a team of scientists from your university, you would all agree that on this planet, the sky is yellow, grass is red and so forth » (ibid., p. 682). Une telle conception « réaliste » des couleurs, et l'interprétation que nous devons en donner dans le contexte de l'argument de Block, soulève certainement des questions intéressantes, mais je me permets de les ignorer et d'admettre cette hypothèse pour les fins de la discussion, même si elle demeure relativement vague et qu'elle porte à controverse. 
À vrai dire, l'expérience de pensée proprement dite invoque une troisième inversion. Comme nous le verrons, l'argument vise à appuyer l'idée d'une inversion fonctionnelle intrapersonnelle, et elle repose sur le scénario suivant que Block nous demande d'imaginer en première personne ${ }^{10}$. Supposons qu'au temps $t_{1}$ je me trouve sur la Terre, disons que je suis étendu dans un hamac sur une plage de la $M$ artinique, par une belle journée où le ciel est d'un bleu limpide. Contemplant la voûte céleste, j'aurais une certaine expérience de couleur, que j'appellerai $E_{1}$, et je serais disposé à énoncer la phrase suivante : «Le ciel est bleu. » On suppose ensuite que je m'endors soudainement et qu'à ce moment une équipe de scientifiques entre en scène pour m'utiliser, à mon insu, comme sujet d'une expérience scientifique. On $m$ 'anesthésie et on $m$ 'insère sous la cornée des lentilles qui ont la propriété d'inverser le spectre des couleurs, de telle sorte que si je me réveillais sur Terre, alors le ciel me paraîtrait jaune, I'herbe rouge et ainsi de suite. C ependant, selon le scénario, je ne me réveille pas sur Terre car pendant mon sommeil les scientifiques auraient fait en sorte que je sois transporté sur Terre inversée, plus précisément dans le hamac où se serait trouvé la contrepartie de moi-même. Ainsi, lorsque je me réveillerais, tout me paraîtrait parfaitement normal : le ciel jaune de Terre inversée me paraîtrait bleu, et ainsi de suite, puisque les lentilles inversantes annuleraient en quelque sorte l'inversion de re qui existe entre les deux environnements. Donc au temps $t_{2}$, je serais sur Terre inversée, sans le savoir, et contemplant la voûte céleste j'aurais une certaine expérience de couleur, que j'appellerai $E_{2}$, et je serais encore une fois disposé à énoncer la phrase : "Le ciel est bleu », ainsi que la phrase : « Le ciel me paraît toujours aussi bleu qu'il me paraissait avant que je ne m'endorme. »

Avant d'aller plus loin, il importe de préciser la stratégie de Block dans son utilisation de cette expérience de pensée. Son but est d'arriver à donner foi à une instance du schéma de thèse suivant :

(T) Deux de mes expériences de couleur, disons $E$ et $E^{\prime}$, qui se produiraient respectivement sur la Terre et sur Terre inversée, auraient le même contenu phénoménal alors qu'elles seraient de types fonctionnels distincts.

La stratégie de Block consiste, premièrement, à suggérer un cas où il serait intuitivement contraignant de penser que deux expériences pertinentes, $E$ et $E^{\prime}$, seraient du même type phénoménal et, deuxièmement, à soutenir

10. J e modifie légèrement le scénario tel que formulé par Block, simplement dans le but de présenter mon argument d'une façon qui soit aussi claire que possible. Cela dit, ma formulation du scénario suit strictement la logique de celle de Block. Par ailleurs, en accord avec une suggestion de Block, je formule le scénario à la première personne, c'est-à-dire en me prenant moi-même comme sujet de l'expérience de pensée. Ce point est important puisque, comme nous le verrons, une des affirmations cruciales de l'argument est que deux expériences de couleur pertinentes seraient du même type phénoménal ; et cette affirmation doit reposer sur des intuitions que nous avons du point de vue de la première personne. 
que dans ce cas nous avons de bonnes raisons de croire que $E$ et $E^{\prime}$ seraient de types fonctionnels distincts. $M$ ais pour bien comprendre son argument, il est important de voir qu'il repose sur les trois présupposés suivants :

(P1) Les expériences de couleur ont un contenu intentionnel.

(P2) Le contenu intentionnel d'une expérience de couleur est déterminé par la signification des prédicats de couleur dans la langue du sujet. (Par exemple, le contenu intentionnel de l'expérience $\mathrm{E}_{1}$ serait déterminé par la signification du prédicat «bleu » en français, car $E_{1}$ me disposerait à produire des énoncés comme « Le ciel est bleu », vrais en français.

(P3) Le contenu intentionnel d'une expérience de couleur détermine son individuation fonctionnelle dans le sens suivant : si deux expériences de couleur ont le même contenu intentionnel (ou différents contenus intentionnels), alors elles sont du même type fonctionnel (ou de types fonctionnels différents).

Pour arriver à trouver deux expériences pertinentes qui permettent d'énoncer une instance vraie du schéma ( $T$ ), Block doit développer davantage son expérience de pensée, car il est tout à fait douteux que les expériences $E_{1}$ et $E_{2}$, telles que décrites plus haut, puissent servir à cette fin, compte tenu des présupposés (P2) et (P3) qui suggèrent que $E_{1}$ et $E_{2}$ seraient du même type fonctionnel, puisqu'il est tout à fait plausible qu'au temps $t_{2}$ mes prédicats de couleur aient la même signification qu'ils avaient à $t_{1}$. L'expérience de pensée doit donc être développée de la façon suivante. Supposons qu'après le temps $t_{2}$ je reste sur Terre inversée pour une période de temps « assez longue ». Et supposons, de plus, que durant cette période, j'interagisse linguistiquement avec les habitants de Terre inversée, d'une façon qu'on pourrait qualifier de normale en ce sens que mes interactions linguistiques se conformeraient aux usages en vigueur, et qu'elles me permettraient de communiquer avec succès. Alors, selon Block, il serait tout à fait plausible qu'après cette période je ne parlerais plus français, mais je parlerais le français inversée ${ }^{11}$. A insi, mes prédicats de couleur n'auraient plus une signification en français, mais une signification en français inversé. Ce point peut être illustré de la façon suivante. Supposons qu'au temps $t_{3}$ je sois resté sur Terre inversée pour une période de temps « assez longue », de sorte que mes prédicats de couleur aient changé de signification. Alors, si j'utilisais les prédicats «bleu », « jaune», et ainsi de suite, à $t_{3}$, ceux-ci auraient dans ma bouche la signification qu'ils ont dans la bouche des locuteurs normaux de Terre inversée. Supposons de plus qu'à $t_{3}$ je me trouve, encore unefois, dans un hamac sur une plage par temps clair, dans des conditions aussi similaires que possibles à celles dans lesquelles j'ai eu

11. Un tel scénario correspond à ce qu'on a appelé des cas de transfert lent (slow switching), qui ont été discutés dans la littérature sur la question de la compatibilité entre l'externalisme sémantique et l'autorité de la première personne. Je crois que Tyler Burge fut le premier à suggérer cette idée, voir Burge, T., «Individualism and Self-K nowledge », Journal of Philosophy, 85, 1988, p. 652. 
I'expérience $E_{1}$. Et supposons, finalement, qu'à $t_{3} j$ 'aie une expérience visuelle, disons $E_{3}$, et que je sois alors disposéà dire : «Le ciel est bleu. »Dans la mesure où par « bleu » je signifierais ce que les habitants de Terre inversée signifient normalement lorsqu'ils utilisent ce prédicat, et compte tenu des présupposés (P2) et (P3), $E_{3}$ aurait un contenu intentionnel différent de celui de $E_{1}$, et par conséquent ces deux expériences seraient de types fonctionnels distincts, alors qu'il est intuitivement contraignant de penser qu'elles auraient le même contenu phénoménal. C'est donc ainsi que Block suggère un exemple qui, sel on lui, satisferait le schéma ( $T$ ).

\section{Un présupposé crucial de l'argument}

Comme je l'ai déjà noté, cet argument est basé sur des intuitions que le lecteur devrait avoir du point de vue de la première personne. En effet, s'il semble intuitivement contraignant d'affirmer que ce que cela fait (what it's like) d'avoir l'expérience $E_{1}$ est similaire à ce que cela fait d'avoir l'expérience $E_{3}$, c'est précisément parce que le lecteur à qui l'on présente l'argument doit être en mesure de concevoir que si lui-même se trouvait dans le scénario, tel que décrit pour le temps $t_{3}$, alors il serait en mesure de faire un jugement de similarité vrai dans lequel il jugerait que ce que cela lui ferait d'avoir l'expérience $\mathrm{E}_{3}$ serait phénoménalement similaire à ce que cela lui aurait fait d'avoir I'expérience $E_{1}$. Autrement dit, me prenant moi-même comme sujet de l'expérience de pensée, je dois être en mesure de concevoir que si je me trouvais dans la situation contrefactuelle décrite, je serais en mesure de dire l'énoncé vrai suivant, au temps $t_{3}$ :

(S) Le ciel me paraît maintenant de la même couleur que dans le passé, il me paraît bleu.

Cepoint semble difficilement contestable. M ais je crois qu'à partir du moment où nous tentons de clarifier les conditions sous lesquelles je pourrais effectivement produire un tel énoncé vrai, cela soulève un sérieux problème pour l'argument de Block, et plus précisément pour l'affirmation selon laquelle $\mathrm{E}_{1}$ et $\mathrm{E}_{3}$ auraient des contenus intentionnels distincts, et que ces expériences seraient de types fonctionnels distincts. Pour clarifier ce quej'ai à l'esprit, il est d'abord utile de reformuler ce point de façon un peu plus précise.

Pour les raisons que je viens de noter, je (en tant que sujet de l'expé rience de pensée) dois être en mesure de concevoir que si je me trouvais dans les conditions décrivant ce qui se passe au temps $t_{3}$, je pourrais exprimer correctement les deux jugements suivants :

(SP) Le ciel me paraît maintenant de la même couleur que dans le passé ;

(P) Le ciel me paraît bleu,

où « maintenant » réfèreà $t_{3}$ et « dans le passé » devrait avoir dans son extension le temps $t_{1}$, ou à tout le moins un certain temps $t^{\prime}$ auquel mes prédicats 
de couleur avaient encore leur signification en français. S'il m'était impossible de concevoir que je puisse faire de tels jugements de similarité vrais - par exemple, si je devais imaginer qu'au temps $t_{3}$ j'aurais perdu toute trace mné monique de ce qui me serait arrivé avant le moment (ou l'intervalle de temps) où mes prédicats de couleurs auraient changé de signification - alors je ne serais aucunement justifié, du point de vue de la première personne, à accepter que ce que cela me fait que d'avoir l'expérience $E_{3}$ serait phénoménalement similaire à ce que cela m'avait fait d'avoir l'expérience $E_{1}$. Sinon, je pourrais tout aussi bien juger « correctement » que ce que cela me fait d'avoir l'expérience $E_{3}$ serait phénoménalement différent de ce que cela m'avait fait d'avoir $E_{1}$. Ce point me semble difficilement contestable. Premièrement, il semble aller de soi que nous pouvons communément faire des jugements comme le suivant : "L'impression que la vision de ce mur me fait, relativement à la couleur, est la même que celle que la vision de cet autre mur m'a fait hier. » $M$ ais s'il nous semble intuitivement plausible que nous puissions aisément faire de tels jugements, qui sont justifiés, cela semble tenir au fait que lorsque nous les faisons nous avons présent à l'esprit, sinon un souvenir exact de ce que nous avons vu, à tout le moins un certain relatum auquel nous comparons notre expérience présente. Car, sinon, ce serait une sérieuse erreur que de considérer de telles expressions comme exprimant un jugement : "A nything would go! ». Or, dans le cas qui nous intéresse, ce relatum doit être un souvenir de l'expérience $E_{1}$ et, dans la mesure où l'argument suppose que $E_{1}$ et $E_{3}$ ont le même contenu phénoménal, il doit s'agir d'un cas où ce souvenir serait fidèle, car si le relatum auquel je comparerais mon expérience présente était d'un type phénoménal distinct de celui de $E_{1}$, alors de deux choses I'une : ou bien mon jugement de similarité serait vrai et le présupposé que $E_{1}$ et $E_{3}$ sont du même type phénoménal serait faux, ou bien ce présupposé serait vrai, et je ne serais pas disposé à faire un tel jugement de similarité, je serais plutôt disposé à nier ce jugement. Q uoi qu'il en soit, l'argument nous oblige à accepter que le sujet devrait être en mesure d'énoncer (SP) et (P), au temps $t_{3}$, et que ces énoncés soient vrais.

$M$ ais si nous devons accepter cela, et si nous devons égal ement accepter la raison offerte par Block pour maintenir que $E_{1}$ et $E_{3}$ sont de types fonctionnels distincts - à savoir qu'au temps $t_{3}$ le sujet ne s'exprimerait plus en français mais en français inversé - alors la question suivante se pose : quelle est la signification de ces phrases en français inversé (et en français)? Et, plus précisément, qu'est-ce que les prédicats « $x$ me paraît dela même couleur que $y$ » et « $x$ me paraît bleu » signifient en français inversé (et en français)? J'aimerais proposer une analyse toute simple de ces prédicats, qui me semble tout à fait acceptable, mais qui a de fâcheuses conséquences pour l'argument de Block. Selon l'analyse que je vais suggérer, le prédicat relationnel « $x$ me paraît de la même couleur que y » $a$, en français inversé, la même signification qu'en français, ce qui n'a rien de très surprenant. $M$ ais, comme je l'explique dans la section suivante, sur la base de cette analyse du prédicat 
relationnel, nous pouvons ensuite produire une analyse du prédicat monadique « $x$ me paraît bleu » à l'aide d'une certaine propriété relationnelle, cequi permet de soutenir que l'énoncé $(P)$, que je serais disposé à énoncer si j'étais dans les conditions décrites au temps $t_{3}$, doit être interprété en français, et non pas en français inversé comme le requiert l'argument de Block.

\section{Pourquoi l'argument doit être rejeté}

Compte tenu de l'expérience de pensée telle que décrite, le prédicat relationnel « $x$ me paraît de la même couleur que y », en français inversé, doit avoir dans son extension, à tout le moins, des paires d'objets que les habitants de Terre inversée s'accordent à catégoriser comme étant de la même couleur lorsqu'ils sont observés dans des conditions normales d'éclairage, par exemple des paires d'objets comme les suivantes : $\left\langle\text { herbe }{ }_{T}, \text { brocoli }_{T 1}\right\rangle_{1}\langle$ citron$1_{\mathrm{Tl}}$, citron- $\left.2_{\mathrm{Tl}}\right\rangle,\left\langle\right.$ ciel $_{\mathrm{Tl}}$, mer $\left._{\mathrm{Tl}}\right\rangle$, etc. (où l'indice «TI » rend explicite qu'il s'agit d'objets de la Terre inversée). De la même manière, des paires d'objets que nous nous accordons à catégoriser comme étant de la même couleur tombent dans l'extension de ce prédicat en français. Si ces deux prédicats avaient dans leur extension uniquement des paires d'objets de Terre inversée et des paires d'objets de la Terre, respectivement, on pourrait dire qu'ils ont des significations différentes, puisqu'ils auraient manifestement des extensions différentes. $M$ ais si c'était le cas, alors le jugement de similarité (SP) ne pourrait être vrai ni en français ni en français inversé, puisqu'une application correcte de ce prédicat, par moi dans l'expérience de pensée, requiert que le prédicat ait également dans son extension des paires d'objets « hétéroclites », c'est-à-dire des paires dont un des deux membres est un objet de la Terre et I'autre un objet de Terre inversée. Ainsi, en plus des paires d'objets notées plus haut, le prédicat, en français inversé, devrait également avoir dans son extension des paires d'objets comme les suivantes: $\left\langle c i e l_{T l}\right.$, citron $\left.\mathrm{n}_{\mathrm{T}}\right\rangle$, $\left\langle\text { herbe }_{T} \text {, tomate }\right\rangle_{1},\left\langle\right.$ citron $_{T}$, mer $\left._{T}\right\rangle$, etc. Et, bien sûr, ces paires d'objets « hétéroclites » devraient également être dans l'extension du prédicat en français. Par conséquent, le prédicat aurait exactement la même extension en français et en français inversé. Cela peut être illustré simplement en imaginant que si les habitants des deux planètes pouvaient voyager régulièrement d'une planète à l'autre, et s'ils faisaient des jugements à propos de la similarité des couleurs de n'importe quelle paire d'objets dans le domaine étendu comprenant les objets des deux planètes, alors leurs jugements de similarité seraient en parfait accord. Ce qui bien sûr ne veut pas dire que leurs attributions de couleurs spécifiques à des objets s'accorderaient. Tout au contraire. Autrement dit, le prédicat monadique « $x$ me paraît bleu » n'aurait pas la même signification dans les deux langues, puisque confronté à un citron n'importe quel locuteur normal du français serait disposé à utiliser le prédicat « jaune » alors qu'un locuteur normal du français inversé serait disposé à utiliser le prédicat « bleu ». 
Cette analyse du prédicat relationnel peut sembler anodine, dans la mesure où elle ne fait que rendre explicite les présupposés sémantiques de l'expérience de pensée, mais elle pose une difficulté pour l'argument de Block. En effet, même si le prédicat relationnel a certaines paires d'objets « hétéroclites » dans son extension, cela ne permet pas de montrer comment le sujet pourrait faire un énoncé vrai en disant (SP) au temps $t_{3}$ car, au contraire, selon cette analyse l'énoncé (SP) serait manifestement faux, qu'il soit interprété en français ou en français inversé, ce qui bien sûr ne fait pas de différence. Cela paraît évident puisque $<c_{i} \mathrm{el}_{\mathrm{T}}$, ciel ${ }_{\mathrm{T}}>\mathrm{n}$ 'est manifestement pas dans l'extension du prédicat. Cette analyse est donc insuffisante, puisqu'il est intuitivement contraignant de penser que le sujet pourrait effectivement exprimer un jugement vrai en énonçant (SP) au tempst ${ }_{3}$. À mon avis, la seule façon de montrer comment cela est possible est tout simplement de modifier l'analyse du prédicat relationnel d'une manière qui tienne compte de la possibilité que les expériences jugées similaires soient produites par la médiation d'un mécanisme d'inversion des couleurs, comme les lentilles inversantes supposées dans l'expérience de pensée. En fait, c'est précisément ce qui semble être à l'œuvre dans notre compréhension du scénario de l'expérience de pensée. L'analyse modifiée est la suivante (notons qu'il est inoffensif de supposer que le prédicat est défini uniquement pour les conditions d'éclairage normales) :

$(R)$ « $x$ me paraît de la même couleur que y » est satisfait, en français inversé, si et seulement si :

i) ni mon expérience de $x, E(x)$, ni mon expérience de $y, E(y)$, n'ont été produites via un mécanisme d'inversion des couleurs, ou toutes les deux l'ont été, et

$$
\begin{aligned}
& <x, y>=\left\{\left\langle\text { herbe } e_{1}, \text { brocoli }_{T 1}>,\left\langle\text { mer }_{T 1}, \text { ciel }_{T 1}>,\langle\text { herbe }\right.\right.\right. \\
& \text { citron } \\
& \text { t tomate } \left._{T 1}>, \ldots\right\}
\end{aligned}
$$

ii) une seule de mes expériences $E(x)$ et $E(y)$ a été produite via un mécanisme d'inversion des couleurs, et

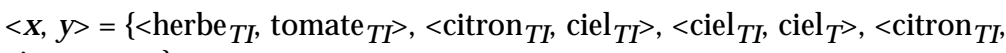
citron $\left._{\mathrm{T}}>\ldots\right\}$

Cette analyse n'a rien de très surprenant. Elle est même plutôt banale, puisqu'elle ne fait que rendre explicite les intuitions sémantiques de l'expérience de pensée. Aussi, il n'est pas étonnant que, selon cette analyse, si j'énonçais (SP) au temps $t_{3}$, cet énoncé serait vrai, et cela sans que nous ayons à supposer ou à nier que j'énoncerais (SP) en français ou en français inversé, puisque l'analyse du prédicat relationnel en français est exactement la même. $M$ ais si nous nous en tenons uniquement à cette analyse du prédicat relationnel - c'est-à-dire si nous faisons abstraction du présupposé (P2) - , elle est insuffisante pour affirmer que les expériences $E_{1}$ et $E_{3}$ ont des contenus intentionnels distincts. En fait, cette analyse et la disposition du sujet à énoncer (SP) suggère plutôt qu'elles auraient le même contenu intentionnel, si 
nous supposons par exempleque deux expériences de couleur ont le même contenu intentionnel si, et seulement si, le sujet est disposéà accepter des jugements les comparant, comme (SP). Et cette suggestion est certainement tout aussi acceptable que le présupposé (P2). II semble donc qu'à ce stade, Block devrait nous donner des raisons de préférer (P2). À mon avis, cela suffit à montrer que l'argument de Block n'est pas du tout contraignant. $M$ ais ce n'est pas tout.

Le problème plus sérieux pour l'argument de Block est de savoir comment $(\mathrm{P})$ devrait être interprété ou, plus précisément, comment le prédicat monadique « $x$ me paraît bleu », tel que je l'utiliserais au temps $t_{3}$, doit l'être. O r, il semble qu'il existe une façon tout à fait naturelle de le faire sur la base de (R). (Ici aussi, il est inoffensif de supposer que le prédicat est défini uniquement pour les conditions d'éclairage normales.) L'analyse est la suivante:

$\left(\mathrm{M}_{\mathrm{FI}}\right)$ « $\mathrm{x}$ me paraît bleu » est satisfait, en français inversé, si et seulement si :

$x$ me paraît être de la même couleur que le ciel $\left.\right|_{\mathrm{Tl}}$ si mon expérience du ciel $\mathrm{Tl}_{\mathrm{I}}$, ou $E\left(\right.$ ciel $\left._{T I}\right)$, est produite d'une façon qui n'implique pas un mécanisme d'inversion des couleurs, ou de la même couleur que le citron ${ }_{T I}$ si mon expérience $E\left(\right.$ citron $\left._{\mathrm{TI}}\right)$ est produite d'une façon qui implique un mécanisme d'inversion des couleurs.

$\left(M_{F}\right)$ « $x$ me paraît bleu » est satisfait, en français, si et seulement si :

$x$ me paraît être de la même couleur que le ciel $T_{T}$ si mon expérience $E\left(\right.$ ciel $\left._{T}\right)$ est produite d'une façon qui n'implique pas un mécanisme d'inversion des couleurs, ou de la même couleur que le citron $\mathrm{T}_{T}$ si mon expérience $E\left(\right.$ citron $_{T}$ ) est produite d'une façon qui implique un mécanisme d'inversion des couleurs.

La première chose importante à noter est que cette analyse repose essentiellement sur le prédicat relationnel tel que défini par (R), mais aussi sur la sélection d'exemplaires pertinents d'expériences auxquels I'expérience définie, soit mon expérience de bleu, est comparée. Deuxièmement, il est utile de se servir des expressions «le ciel $\left.\right|_{T I} »$, « le citron ${ }_{T I}$ », « le ciel $\left.\right|_{T}$, et «le citron $n_{T} »$ pour formuler simplement ces conditions, mais rien d'essentiel ne repose sur cette façon de procéder. D'autres termes singuliers feraient tout aussi bien I'affaire. En fait, il serait peut-être même préférable de penser que les exemplaires pertinents doivent être sélectionnés de façon démonstrative. L a seule contrainte est que ces termes singuliers sélectionnent les ex emplaires de telle sorte que « $x$ me paraît bleu » signifie en français inversé ce que « $x$ me paraît jaune » signifie en français, et inversement ${ }^{12}$. Finalement, il semble

12. Cette contrainte sur le choix des termes singuliers servant à sélectionner les exemplaires pertinents peut être précisée de la façon suivante. Supposons que $a_{1}, a_{2}, a_{3}, a_{4}$, sont les termes qui jouent respectivement les rôles de « le ciel $\left.\right|_{T l}$ », «le citron $n_{T l}$ », «le ciel ${ }_{T}$ », et «le citron $n_{T}$ » dans les conditions $\left(M_{F I}\right)$ et $\left(M_{F}\right)$. Ces termes doivent être tels que des locuteurs normaux du français et du français inversé s'accorderaient à dire que le prédicat « $x$ est de la même couleur que y » i) est satisfait par $\left\langle a_{1}, a_{4}\right\rangle$ et $\left\langle a_{2}, a_{3}\right\rangle$ (et symétriquement), et ii) qu'il n'est satisfait par aucune des paires suivantes: $\left\langle a_{1}, a_{2}\right\rangle,\left\langle a_{3}, a_{4}\right\rangle,\left\langle a_{1}, a_{3}\right\rangle,\left\langle a_{2}, a_{4}\right\rangle$ (et symétriquement). 
évident que, selon cette analyse, « $x$ me paraît bleu » a des extensions différentes en français et en français inversé.

C ette analyse semble tout à fait acceptable. Encore une fois, elle ne fait que rendre explicite les intuitions sémantiques présupposées dans notre compréhension de l'expérience de pensée ${ }^{13}$. $M$ ais elle semble être en conflit avec la raison que Block propose pour appuyer l'idée que $E_{1}$ et $E_{3}$ auraient des contenus intentionnels différents, à savoir qu'au temps $t_{3}$ le sujet s'exprimerait en français inversé et non pas en français. La raison de ce conflit est que si nous acceptons cette analyse, et si nous acceptons également qu'au temps $t_{3}$ le sujet doit pouvoir énoncer $(P)$ et que cet énoncé serait vrai, alors il semble que le sujet devrait s'exprimer en français, et non pas en français inversé. Supposons que j'énonce $(P)$ au temps $t_{3}$. Alors cet énoncé pourrait difficilement impliquer que le ciel ${ }_{T}$, tel que je l'observerais au temps $t_{3}$, me paraîtrait de la même couleur que le ciel $\mathrm{T}_{\mathrm{T}}$ me paraîtrait si mon expérience $E\left(c_{i e l}\right)$ était produite d'une façon qui n'implique pas un mécanisme d'inversion des couleurs. Et, il n'impliquerait pas plus que le ciel ${ }_{T}$, tel que je l'observerais au temps $t_{3}$, me paraîtrait de la même couleur que le citron $n_{T I}$ si mon expérience $E\left(\right.$ citron $_{T}$ ) était produite d'une façon qui implique un mécanisme d'inversion des couleurs. $M$ ais, si j'énonçais $(P)$ en français inversé, il découle logiquement que l'une ou l'autre de ces conséquences devrait être vraie.

Le point est tout simplement que s'il nous semble intuitivement contraignant d'accepter l'idée que $\mathrm{E}_{1}$ et $\mathrm{E}_{3}$ ont le même contenu phénoménal, c'est précisément parce que nous comprenons que ces deux expériences disposeraient le sujet à produire deux énoncés vrais de « Leciel me paraît bleu » (ou « Ceci me paraît bleu ») dans lesquels il aurait l'intention d'utiliser «me paraît bleu » dans un seul et même sens! Et ce sens ne peut être que celui que nous donnons à ce prédicat en français. Ainsi, il est absurde de dire qu'au temps $t_{3}$ le sujet utilise ce prédicat monadique en français inversé, du moins si celui-ci doit être entendu dans le sens quej'ai proposé, ce qui me semble difficilement contestable puisque mon analyse n'est qu'une explicitation des intuitions sémantiques qui nous permettent de comprendre l'expérience de pensée. De plus, si l'énoncé $(P)$ est interprété comme étant en français, en accord avec l'analyse notée plus haut, il devient non seulement cohérent mais vrai. Car, en effet, cet énoncé semble bien impliquer que le ciel $_{T I}$, tel que je l'observerais au temps $t_{3}$, me paraîtrait de la même couleur que le ciel ${ }_{T}$ si mon expérience $E\left(\right.$ ciel $\left._{T}\right)$ était produite d'une façon qui n'implique pas un mécanisme d'inversion des couleurs, et que le ciel $_{\mathrm{Tl}}$, tel que je l'observerais

13. Cette analyse semble être en accord avec l'esprit de l'analyse des prédicats de couleur proposée par W ilfrid Sellars dans son article «Empiricism and the Philosophy of M ind », dans Feigl, $H$. et Scriven M ., dir., M innesota Studies in Philosophy of Science, vol. 1, M inneapolis, University of $M$ innesota Press, 1956, récemment réédité dans Sellars, W., Empiricism and the Philosophy of M ind, Cambridge (M ass.), H arvard University Press, 1997. 
au temps $t_{3}$, me paraîtrait de la même couleur que le citron $n_{T}$ si mon expé rience $E\left(\right.$ citron $\left._{\mathrm{TI}}\right)$ était produite d'une façon qui implique un mécanisme d'inversion des couleurs. C'est pourquoi nous devons rejeter l'affirmation, cruciale à l'argument de Block, selon laquelle les expériences $E_{1}$ et $E_{3}$ auraient des contenus intentionnels différents, et donc qu'elles seraient de types fonctionnels distincts. $M$ on argument suggère que ces expériences auraient bel et bien le même contenu intentionnel et qu'elles seraient du même type fonctionnel.

Avant de conclure, il me paraît utile de reformuler mon argument de façon un peu plus systématique, et cela sur la base d'un principe général qui rend explicite une relation conceptuelle importante entre le prédicat relationnel « $x$ me paraît de la même couleur que $y$ » et le prédicat monadique « $x$ me paraît bleu ». Ce principe systématise tout simplement l'idée suivante. Q uelles que soient les paires d'objets qui satisfont le prédicat relationnel dans une langue $L$, les objets dans ces paires devraient être tels que si l'un d'eux satisfait le prédicat monadique, I'autre doit le satisfaire également. ( $D$ ans ce qui suit j'utilise « Sxy » pour représenter le prédicat relationnel « $x$ me paraît de la même couleur que $y$ », et « $B x$ » pour représenter le prédicat monadique « $x$ me paraît bleu ».) Le principe général peut être formulé de la façon suivante :

$$
\text { (PG) } \forall(x) \forall(y)(S x y \supset(B x \equiv B y))
$$

Ce principe semble très plausible et difficile à nier. Et, comme je l'ai proposé, il semble que nous devons admettre qu'au temps $t_{3}$, le sujet serait en mesure d'énoncer (SP) et (P) et que ces énoncés seraient vrais. Sur cette base nous pouvons formuler le raisonnement suivant :

1) B ciel $_{T 1}$ de (P)

2) $S\left(\right.$ ciel $_{T l}$, ciel $\left._{T}\right)$ de (SP)

3) B ciel $_{\mathrm{T}}$ de 1, 2, et (PG)

4) Bciel $_{T I} \&$ Bciel $_{T}$ de 1 et 3

Or, dans la mesure où le principe (PG) est intuitivement contraignant, il semble que ce soit là un raisonnement que le sujet de l'expérience de pensée pourrait facilement faire au temps $t_{3}$. M ais il est évident que si la ligne 4 était interprétée en français inversé, selon l'analyse que nous avons suggérée plus haut, cet énoncé serait incohérent. $M$ ais il est parfaitement cohérent s'il est interprété en français, en accord avec $\left(\mathrm{M}_{\mathrm{T}}\right)$.

Un fonctionnaliste semble donc être dans une excellente position pour rejeter la raison offerte par Block à l'appui de l'idée que nous pourrions facilement imaginer une instance vraie du schéma $(T)$, puisqu'il ne nous a donné aucune bonne raison de croire que les deux expériences pertinentes, soit $E_{1}$ et $E_{3}$, seraient de types fonctionnels différents. D e plus, bien que mon argument présuppose (P1) et (P3), les considérations quej'ai évoquées suggèrent que le présupposé (P2) serait trompeur, dans la mesure où il semble que ce ne soit 
pas tant la signification des prédicats de couleur qui détermine le contenu intentionnel des expériences de couleurs, ou à tout le moins pas directement, mais que celui-ci est déterminé plutôt par la signification de prédicats ayant la forme « $\mathrm{x}$ me paraît $\mathrm{C}$ » (où « $C$ » doit être remplacé par un prédicat de couleur).

Selon Block, son argument permettrait d'établir l'existence d'une distinction substantielle entre le contenu intentionnel des expériences sensorielles et leur contenu purement phénoménal. $\mathrm{M}$ ais si mon argument est correct, cette distinction est trompeuse et nous devons la rejeter. Comme je l'ai noté dans la première section, la principale motivation de Block est de proposer un argument qui ne serait pas confronté à certaines supposées objections d'incohérence auxquelles l'argument du spectre inversé serait confronté, dans sa version intrapersonnelle classique. Bien qu'il ne me paraisse pas évident que ces objections d'incohérence soient fondées, comme je l'ai noté plus haut, et bien que mon objectif ici n'est pas de les évaluer, il me semble qu'il serait utile de discuter cette question à la lumière des considérations que j'ai proposées. M ais c'est là une tâche que je dois laisser pour une autre occasion. Q uoi qu'il en soit, une chose est claire : si mon argument est correct, alors il n'y a aucune raison de penser que l'argument de la Terre inversée devrait être préféré à l'argument classique du spectre inversé, que ce dernier soit ou non confronté à des objections d'incohérence ${ }^{14}$.

14. Je remercie R enée Bilodeau, M urray Clarke, $\mathrm{N}$ icholas Kaufmann, Daniel Laurier, Brian M CLaughlin, Kai N ielsen, Claude Panaccio, et Richard Vallée pour leurs commentaires sur des versions antérieures de ce texte. 